\title{
Electrons on the HDice target: Results and analysis of test runs at JLab in 2012
}

\author{
M. M. Lowry ${ }^{\star a}$, C. D. Bass ${ }^{a}$, A. D’Angelo ${ }^{b}$, A. Deur ${ }^{a}$, C. Hanretty ${ }^{c}$, D. Ho ${ }^{d}$, T. Kageya ${ }^{a}$, \\ V. Laine ${ }^{a, e}$, P. Peng ${ }^{c}$, A. M. Sandorfi ${ }^{a}$, X. Wei ${ }^{a}$, and C. S. Whisnant ${ }^{f}$ \\ ${ }^{a}$ Thomas Jefferson National Accelerator Laboratory, 12000 Jefferson Ave., Newport News, VA \\ 23606, USA \\ ${ }^{b}$ Universita' di Roma Tor Vergata and INFN Roma Tor Vergata, Via della Ricerca Scientifica, 1 \\ 00133 Rome, Italy \\ ${ }^{c}$ University of Virginia, Charlottesville, VA 22903, USA \\ ${ }^{d}$ Carnegie Mellon University, Pittsburgh, PA 15213, USA \\ e Blaise Pascal University, 63170 Aubière, France \\ $f$ James Madison University, 901 Carrier Dr., Harrisonburg, VA 22807,USA \\ E-mail: mlowry@jlab.org, bassc@lemoyne.edu, \\ annalisa.dangelo@roma2.infn.it, deurpamejlab.org, \\ hanrettyejlab.org, daohejlab.org, kageya@jlab.org, laine@jlab.org, \\ peng@jlab.org, sandorfi@jlab.org, xwei@jlab.org, whisnacs@jmu.edu
}

\begin{abstract}
During the Jefferson Labaratory E06-101 (g14) experiment [1] utilizing photons on solid HD and performed in Hall B, two opportunities arose for targets to be subjected to multi-GeV electron beams in week-long campaigns of dose accumulation and NMR polarization measurements. This was in preparation for conditionally approved electron experiments after the $12 \mathrm{GeV} \mathrm{JLab}$ upgrade[2]. Besides the important thermal effects, evidence consistent with screening of the NMR and with decay of the target polarization was observed during bombardment and for a time afterwards. The solid hydrogens have been the subject of previous radiation damage studies, both for possible polarized DT fusion[3] and for production of dynamically polarized nuclear targets[4]. We synthesize all this information into an overall picture that can guide on-going development of the HDice target system for future use.
\end{abstract}

XVth International Workshop on Polarized Sources, Targets, and Polarimetry

September 9 - 13, 2013

Charlottesville, Virginia, USA

\footnotetext{
* Speaker.

${ }^{\dagger}$ Current address: Le Moyne College, Syracuse, NY 13214, USA
} 


\section{Introduction}

The HDice frozen-spin target was utilized in the E06-101 (g14) experiment [1] with polarized photons as one of the last experiments of the $6 \mathrm{GeV}$ era at Jefferson Laboratory. Twice during the course of the run, the beam was switched to electrons and week-long sequences of irradiation and NMR measurements were done. The target is proposed for use with electrons in two experiments in the $12 \mathrm{GeV}$ era [2] and more information is required as to its survivability and possible enhancement than the initial tests of Mano and Honig[5]. After an overview of the target system, we will describe cryostat temperature changes observed and relate them to energy deposition, beam rastering and target cooling calculations. The NMR measurement results are then reported and interpreted in terms of electron beam heating, possible mechanisms for polarization loss, and the irradiation induced chemistry. Finally, the plans for future measurements and the prospects for use of HD in electron beam experiments will be discussed.

\section{HDice Target Overview}

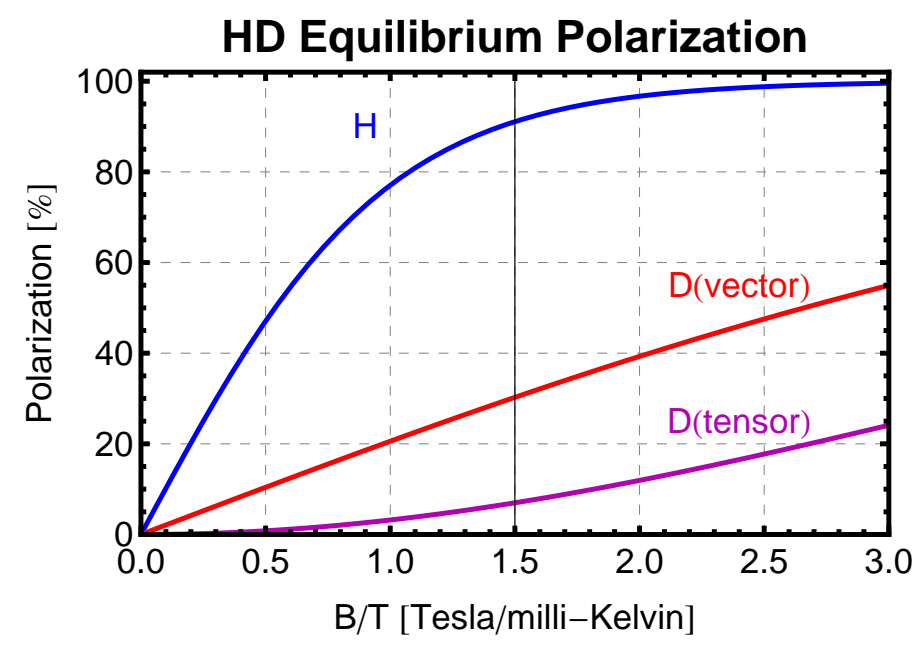

Figure 1: Equilibrium vector and tensor polarizations of $\mathrm{H}$ and $\mathrm{D}$ versus $\mathrm{B} / \mathrm{T}$ ratio. The vertical line at 1.5 $\mathrm{T} / \mathrm{mK}$ corresponds to $\mathrm{B} / \mathrm{T}$ for polarizing conditions of 15 Tesla and $10 \mathrm{mK}$ but polarizations achieved in practice correspond to a ratio of about $0.8 \mathrm{~T} / \mathrm{mK}$.

First proposed 46 years ago [6], the current incarnation of a solid HD frozen-spin target, named HDice, has been described before[7]. The HDice target consists of a solid cylinder of frozen hydrogen deuteride. It is polarized by placing the target in a dilution refrigerator with base temperatures below 10 milli-Kelvin and with a magnetic field of 15 Tesla. As Figure 1 shows, the equilibrium polarizations for the two species in these conditions are about $90 \%$ and $30 \%$ vector polarization for $\mathrm{H}$ and $\mathrm{D}$, respectively. However, pure HD has a multi-year T1 under these conditions so polarizing it is impractical. This problem is solved by the presence of small $\left(\sim 10^{-4}\right)$ admixtures of ortho-H2 and para-D2. (For more on target material preparation see [8].) Because of fermionic and bosonic symmetry constraints, these species are meta-stable (mean lifetimes $=6.3$ and 18.6 days for $\mathrm{H}$ and $\mathrm{D}$, respectively). Under polarizing conditions, they retain one unit of molecular rotation 


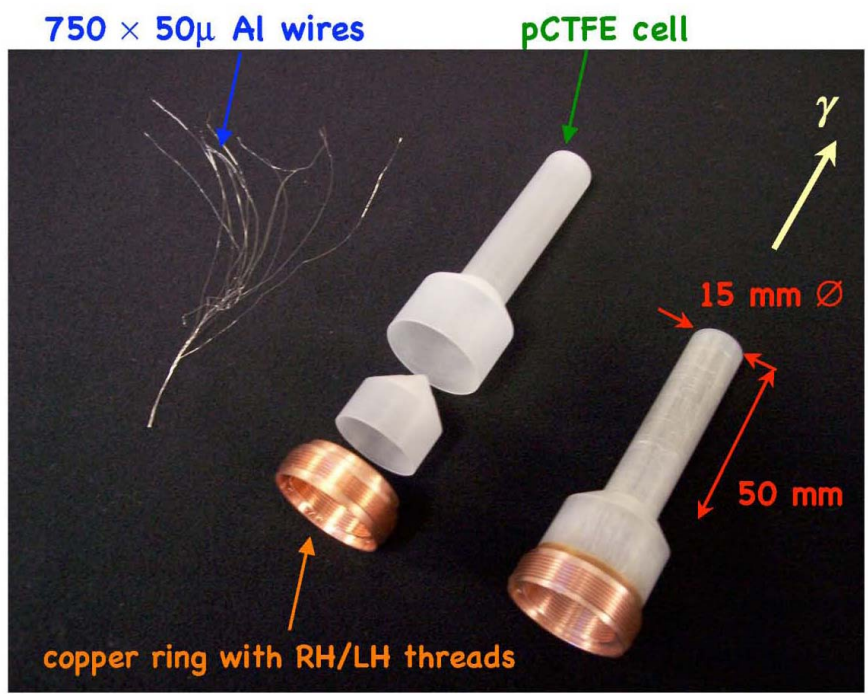

Figure 2: Components of the HDice target cell, showing one of the 60 bundles of wire on the left, the pCTFE shells and copper ring in the center, and the assembled cell on the right.

and hence an atomic quadrupole moment that couples nuclear spins to the lattice temperature. By spin diffusion, the resulting polarization is spread throughout the target. Further, the meta-stable decay means that after sufficient waiting time, the long T1 of pure HD is restored and the target can be moved for use in less extreme conditions of temperature and field. Unfortunately, the decay also produces heating of the target. Although we introduce aluminum cooling wires to carry off the heat, the target temperature is raised above the refrigerator base temperature so the polarization achieved is a competition between the heating reduction and $\mathrm{T} 1$ growth as the metastables disappear. In practice, both $\mathrm{H}$ and $\mathrm{D}$ freeze out at around a $\mathrm{B} / \mathrm{T}$ ratio of $\sim 0.8 \mathrm{~T} / \mathrm{mK}$.

The target also offers the opportunity to do RF manipulations on the polarizations to rotate and transfer spin. (For more on this option see [9].)

The least extreme conditions we use are in a transfer cryostat with field of 0.12 Tesla and 1.9 $\mathrm{K}$ [10], which is used to move the target out of the polarizing dilution refrigerator, into a transport cryostat at 2 Tesla and 2.1 Kelvin for the trip to the experimental hall, and then into an in-beam cryostat. The last device is a dilution refrigerator with a base of $50 \mathrm{mK}$ and a holding field of 1 Tesla.

The target geometry chosen for photon running and shown in Figure 2 was a $15 \mathrm{~mm}$ diameter right cylinder, $50 \mathrm{~mm}$ long, encased in a poly-CTFE shell and cooled by either 750 50-micron or 96038 -micron aluminum wires. The wires were soldered into a copper ring that had inner lefthanded threads for attachment to the transfer cryostat and outer right-handed threads for attachment to any of the other cryostats. By mass, the material in the beam is $77 \% \mathrm{HD}, 17 \% \mathrm{Al}$ and $6 \%$ pCTFE.

\section{HDice Target Temperature}

The top panel of Figure 3 shows the temperature rise of the mixing chamber of the in-beam 


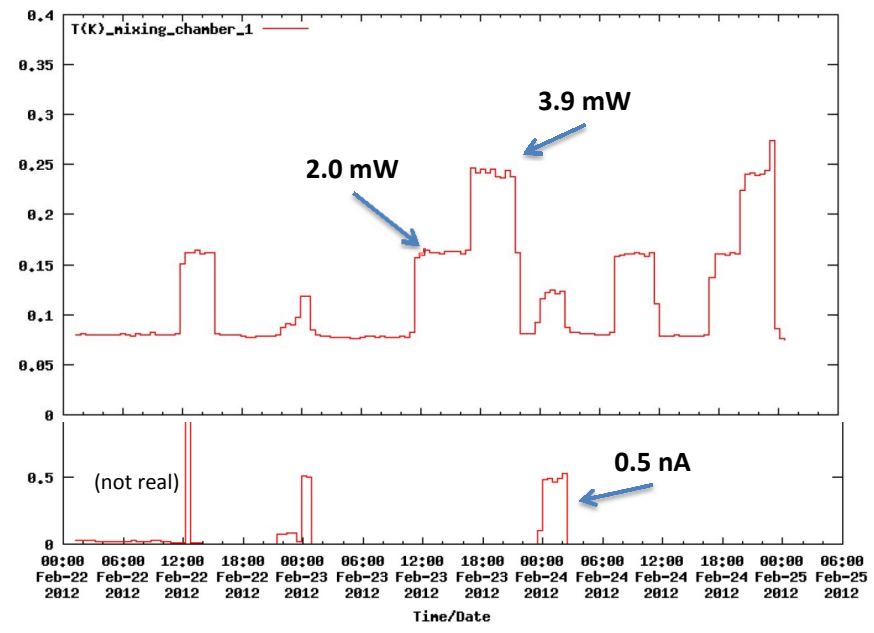

Figure 3: IBC mixing chamber temperature (top) and electron beam current (bottom) during the first set of tests. Applied heater powers indicated in top panel.

\begin{tabular}{|c|c|c|c|}
\hline \multicolumn{5}{|c|}{ Thermal Resistivities $(\rho)$ Al from Ref. [11], HD from Ref. [12] } \\
\hline Temperature [K] & $\mathrm{Al}[\mathrm{m}-\mathrm{K} / \mathrm{W}]$ & $\mathrm{HD}[\mathrm{m}-\mathrm{K} / \mathrm{W}]$ & Kapitza $\left[\mathrm{m}^{2}-\mathrm{K} / \mathrm{W}\right]$ \\
\hline 0.800 & 0.00208 & 1.14 & 0.00095 \\
\hline 0.200 & 0.00833 & 71.3 & 0.0608 \\
\hline 0.020 & 0.0833 & 71250 & 60.8 \\
\hline \multicolumn{5}{|c|}{ Characteristic Geometry -960 38-micron wires, $15 \mathrm{~mm}$ dia. x 50mm long cell } \\
\hline \multicolumn{5}{|c|}{$\mathrm{Al}$} & $\mathrm{HD}$ & Kapitza \\
\hline Area $\left[\mathrm{m}^{2}\right](A)$ & $1.1 \times 10^{-6}$ & $5.7 \times 10^{-3}$ & $5.7 \times 10^{-3}$ \\
\hline Length $[\mathrm{m}](L)$ & 0.05 & $2.2 \times 10^{-4}$ & - \\
\hline \multicolumn{4}{|c|}{ Thermal Resistances $-R=\rho \times L / A$} \\
\hline Temperature $[\mathrm{K}]$ & $\mathrm{Al}[\mathrm{K} / \mathrm{W}]$ & $\mathrm{HD}[\mathrm{K} / \mathrm{W}]$ & Kapitza $[\mathrm{K} / \mathrm{W}]$ \\
\hline 0.800 & 95.2 & 0.044 & 0.165 \\
\hline 0.200 & 381 & 2.77 & 10.57 \\
\hline 0.020 & 3810 & 2770 & 10570 \\
\hline
\end{tabular}

Table 1: Thermal Conduction in the HD target. 


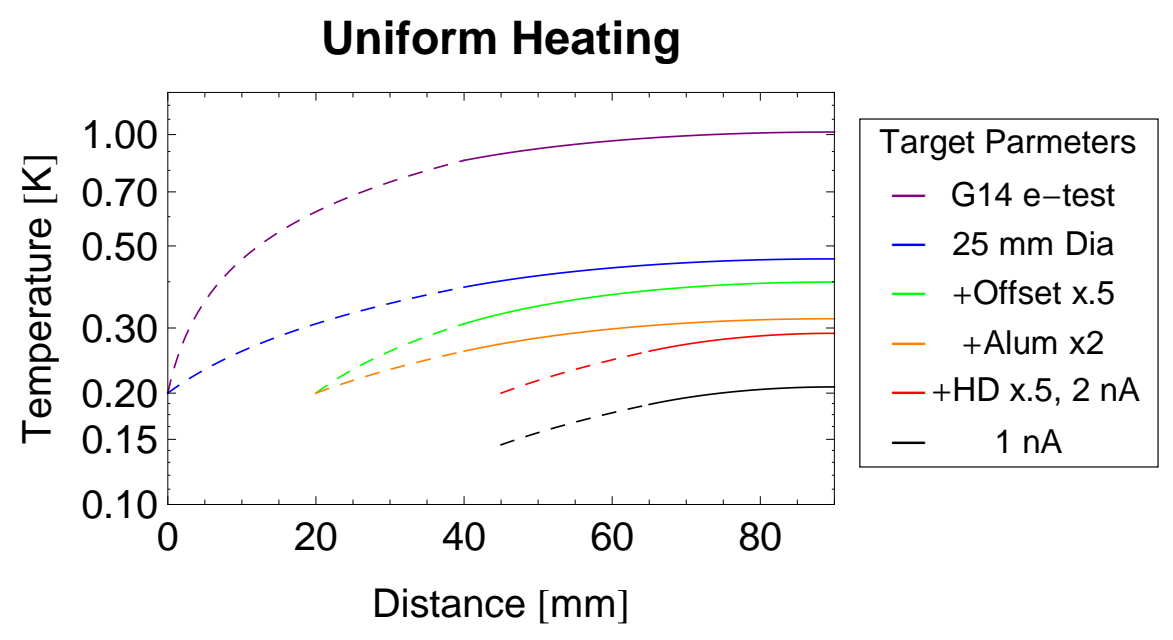

Figure 4: HD target temperature distribution along the beam axis for uniform heating by an electron beam. The copper ring attachment to the mixing chamber is on the left. The solid portion of the curves indicates where the wires are immersed in the HD while the dashed section is in vacuum. The top, violet, curve corresponds to the 2012 electron tests, with $1 \mathrm{nA}$ of beam and a $15 \mathrm{~mm}$ diameter target and shows a target temperature around 1 Kelvin. The blue curve shows the reduction when the same beam is spread over a 25 $\mathrm{mm}$ diameter. The green curve additionally moves the copper ring a factor of two closer to the HD. The orange curve adds twice the density of aluminum wires. The red curve further cuts the length of the target a factor of two and doubles the beam current to keep the energy deposition constant. The bottom, black, curve drops the current back to $1 \mathrm{nA}$, also changing the heat load on the IBC and thus dropping the copper ring temperature. These changes culminate in a 5-times-lower average target temperature.

cryostat, both when subject to heating from a resistor for cooling power determination and during bombardment of the target by a $0.5 \mathrm{nA}$ multi-GeV electron beam, whose current is shown in the bottom panel. The temperature rise is consistent with calculated energy deposition of $0.5 \mathrm{~mW} / \mathrm{nA}$ $\mathrm{cm}$ on this $4 \mathrm{~cm}$ long target. However, to determine the temperature of the HD and aluminum wires, we must rely on thermal conduction calculations of the cooling through the HD and down the aluminum wires. The ingredients of that calculation can be seen in Table 1. Although the thermal resistivity of the HD is the highest at all temperatures, it makes the smallest contribution to the thermal resistance because of the characteristic geometry differences. For the temperaure regime relevant to irradiation, 0.2-1.0 Kelvin, the target temperature distribution is detemined by the aluminum wires. The results of exact differential equation solutions are shown in Figure 4. The $\mathrm{HD}$ and imbedded wires averaged about $1 \mathrm{~K}$ during the electron tests with $1 \mathrm{nA}$ of beam, top violet curve in Figure 4. Simple changes in the target geometry will allow it to cool down to $0.2 \mathrm{~K}$ with the same beam current, bottom black curve in Figure 4.

These results are for a beam rapidly and uniformally swept over the target cross-section. The existing Hall B raster was only marginal in this regard. It has a minimum sweep time (minimum radius to maximum and back) of 1 second whereas the target geometry used had a thermal response time of 0.4 seconds. The problem will be worse once the improved cooling is implemented, thermal response time will be about 2 milli-seconds. A $1 \mathrm{KHz}$ raster has been designed for future operations and is being constructed. 


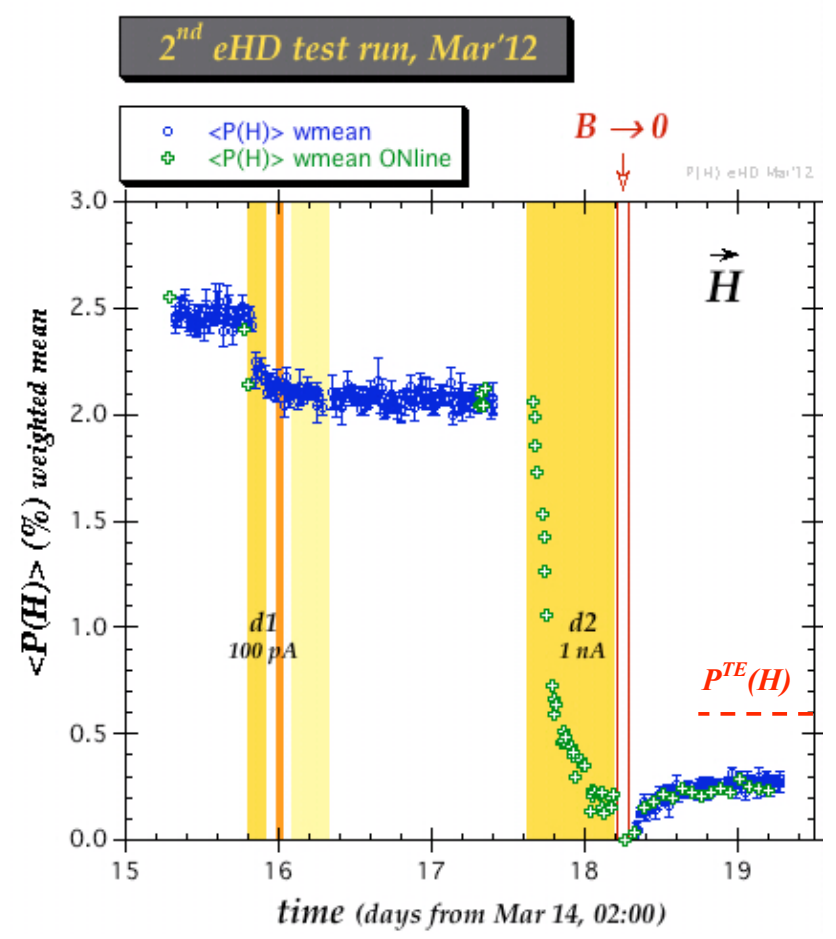

Figure 5: NMR measurements during the second test run in 2012.

\section{HDice Target NMR Measurements}

Crossed NMR coils are built into the in-beam cryostat and allow monitoring of the $\mathrm{H}$ and D polarizations. During the first set of electron beam tests, measurements were taken with these coils following short irradiations. No consistent, permanent change in the hydrogen T1 was found but the situation was confused by attempts to map out field and temperature dependences and by the low polarization of the target. There was evidence for significant short term screening of the RF fields, presumably by free electrons liberated by the beam, and it was clear that most of the polarization was lost during the irradiation by the beam.

Figure 5 shows the NMR amplitude during the second test run. NMR scans were done every 15 minutes during the irradiation. As can be seen in the bombardment period labeled "d2", there is an initial fast amplitude decay of roughly $50 \%$ followed by a slower decay with time constant of 4 to 6 hours. Following the beam-on period, the magnetic field was set to zero for about an hour and then the field was raised again. The polarization started to grow in but became frozen at a value below equilibrium. The initial loss can be explained in terms of RF shielding developing as the bombardment generates free electrons that reach an equilibrium concentration after a few hours. Polarization loss then becomes visible with a time constant of 4 to 6 hours. The interrupted polarization re-growth has a similar time constant. Thus we find a depolarizing irradiation damage that self anneals post-irradiation. Note that the self anneal presumably also occurs durring irradiation so that the depolarizing damage builds to some equilibrium level and remains there rather than continuing to build up. 


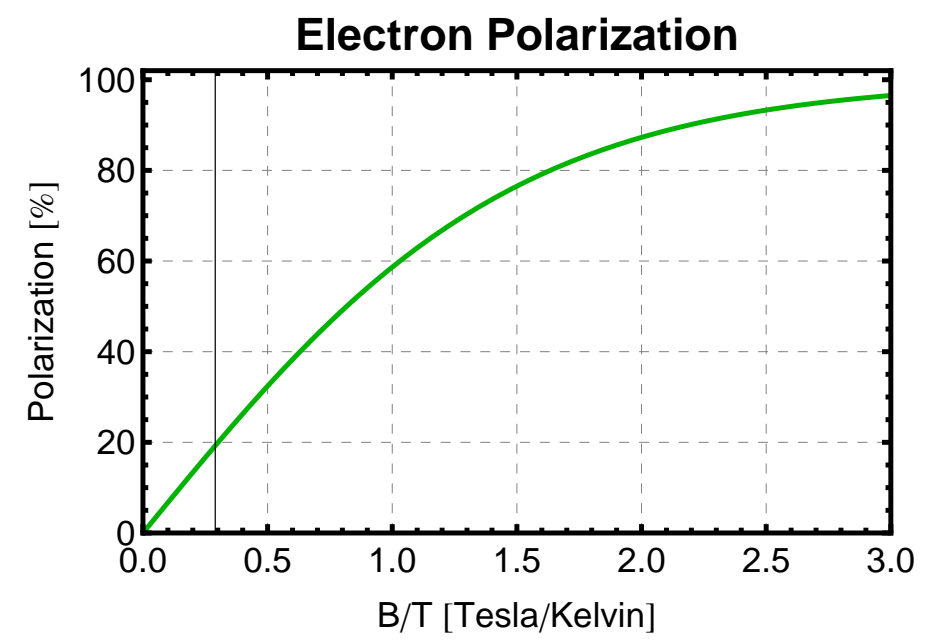

Figure 6: Electron Polarization. The thin vertical line marks the B/T value for the second run, giving an electron polarization of $20 \%$.

\section{Polarization relaxation mechanisms}

We have considered two depolarization mechanisms that may be responsible here. Both result from un-paired bound electron spins. The first arises because an unpaired spin represents a paramagnetic impurity following the theory of Rorschach[13]. The un-paired electron flips its spin as a result of interactions with lattice phonons so as to equilibrate the electron spin temperature to the lattice. The flips produce a time varying field with a component at the Larmour frequency of the nuclear spin. Occasionally this electron flipping induces a nuclear flip that depolarizes a nearby nuclear spin and then spin diffusion spreads the depolarization to the rest of the target.

As Figure 6 shows, the electron polarization for the second run was only about 20\%, so one obvious improvement would be to raise that polarization, resulting in fewer electron spin flips. For example, with a 1 Tesla holding field and at the $0.2 \mathrm{~K}$ of the bottom curve in Figure 4, the electron polarization is $99.8 \%$. At the same time, the higher electron polarization raises the average field in the vicinity of the paramagnetic impurity, inhibiting spin diffusion. Finally, raising the holding field will increase the nuclear resonance frequency and thus require a higher frequency flip field component with a smaller amplitude.

The second mechanism for depolarization is the hyperfine interaction. This mixes the individual angular momenta of the electron and the nucleus while preserving the total angular momentum. Thus the individual m-values are no longer good quantum numbers, the nucleus has an admixture of the wrong direction which can spin diffuse to the rest of the target and depolarize it. Note that using a higher field will suppress the mixing. A higher electron polarization will impede spin duffusion as above. The mixing is eliminated in the "stretched" angular momentum sub-state, which requires, assuming a positive nuclear moment, that the nuclear polarization be opposite the field. This can be accomplished with the RF procedures discussed in [9]. In that case, only the anti-aligned electrons contribute to hyperfine depolarization.

Improving the $\mathrm{B} / \mathrm{T}$ ratio should have an effect on both these processes. We hope to go from $0.29 \mathrm{~T}$ to $1.0 \mathrm{~T}$ and from $1.0 \mathrm{~K}$ to $0.2 \mathrm{~K}$. That will reduce the anti-aligned electron moment pop- 


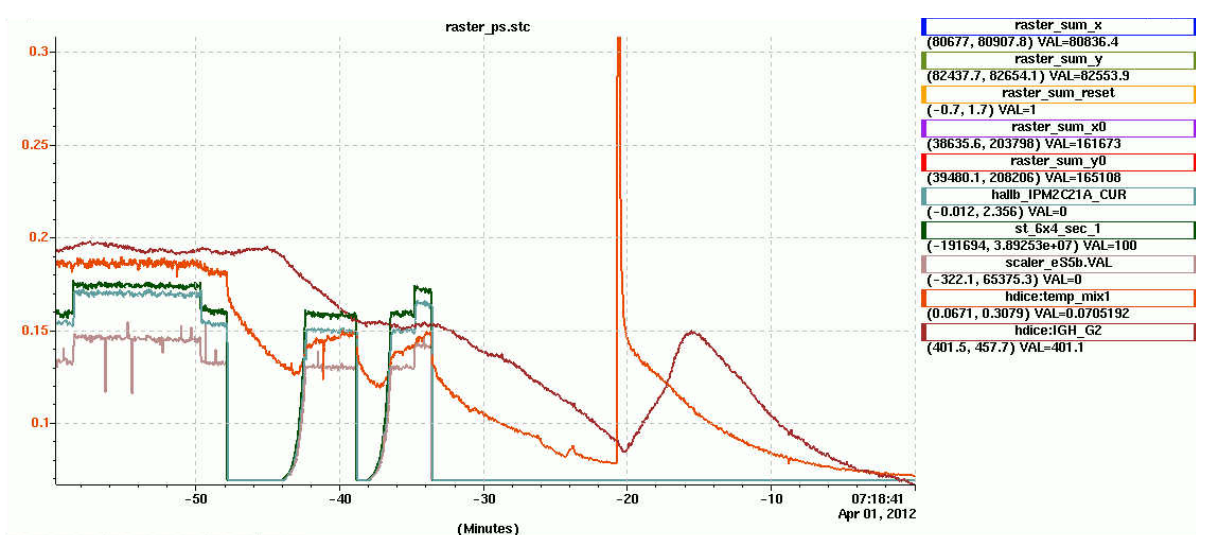

Figure 7: Online strip chart showing the beam current in green, the mixing chamber temperature in light red, and the dilution refrigerator return pressure in dark red versus time in minutes. Note the only roughly $20 \%$ immediate drop in power when the beam is lost at -48 minutes and the sudden temperature rise, presumably due to a spontaneous recombination flash, at -20 minutes. The event clearly deposits additional energyas it is also accompanied by additional ${ }^{3} \mathrm{He}$ being driven out of the mixing chamber and causing a temporary rise in the return pressure.

ulation by a factor of about 400 and a factor of 400 would transform the 4 to 6 hour lifetime into 2 to 3 months. This is a much more useful regime for a frozen spin target.

\section{Irradiation chemistry}

Study of irradiation effects on molecular hydrogen has a 60 year history because of the efforts to understand tritium decay, the desire to polarize tritium for inertial fusion [3] and the interest in a dynamically polarized solid hydrogen target [4]. From early gaseous hydrogen studies, the energy deposited per ion pair equals $36.3 \mathrm{eV}$ [14] and the number of atoms per ion pair is $5.1 \pm 0.3$ [15]. The ionization threshold is $15 \mathrm{eV}$ and the disassociation threshold is $11 \mathrm{eV}$ with a $4.5 \mathrm{eV}$ energy cost. The reason the energy works out is because the $H_{2}^{+}$ion is extremely reactive. It rapidly grabs another $\mathrm{H}$ from a nearby $\mathrm{H}_{2}$ to form an $\mathrm{H}_{3}^{+}$ion and an atomic $H$. (Note the use of $\mathrm{H}$ here to designate either isotope.)

Thus we have three species initially produced by the irradiation. Free electrons form polarons which may become localiced on lattice defects. Because of their large zero point motion, they form a large bubble with accompanying lattice distortion, and are thereby fairly immobile. The $\mathrm{H}_{3}^{+}$ ion has two paired electrons and should not be a source of de-polarization. It is a small triangle with relatively high mobility. (It does occassionally form $H_{9}^{+}$clusters and become trapped.) The third species, atomic hydrogen, is the main concern for the depolarization mechanisms already discussed. Atomic hydrogen has an intermediate mobility and direct re-combination is thought to be very slow.

Reactions involving the free electron can also occur. The $H_{3}^{+}$ion may capture a free electron to generate an $H_{2}$ and an atomic $H$, although $3 H$ 's are also energetically possible. Once the concentration of atomic $H$ is high enough, capture of an electron to form an $H^{-}$ion will become important. This species is immobile but if a wandering atomic $H$ should encounter it, they will rapidly recombine and eject the electron. The electron is then free to be captured by another atomic 
$\mathrm{H}$ and repeat the process. (Note that the $\mathrm{H}_{2}^{-}$ion is not stable in vacuum.) Further the $4.5 \mathrm{eV}$ released by recombination is sufficient to free another electron or enhance the mobility of atomic $H$, possibly leading to a chain reaction. Such recombination flashes were first seen by Webeler [16] in tritium doped solid hydrogen samples maintained between 0.2 and $0.8 \mathrm{~K}$. Figure 7 shows an example of such a flash in our measurements.

Webeler's results have another feature in common with our results. He observed that more than $75 \%$ of the deposited energy did not appear initially and had presumably gone into molecular dissociation [17]. This observation was confirmed by anomalously large EPR signals seen by polarized fusion researchers. We observed a gradual build up to full energy over roughly 15 minutes when beam was first applied and, when bombardment ended, an immediate drop of about $20 \%$ followed by a slow fall off in power over about 20 minutes. In order for so much of the deposited energy to be tied up in broken molecular bonds three times as many atoms must be produced in the solid as in the gas. The fact that we saw a build up to the predicted power implies that a steady state concentration of atoms is being reached, with a balance of dissociation and recombination. That, in turn, implies that the atom-induced depolarization rate also reaches an equilibrium value and does not continue to grow with dose.

However, there is one problem that does grow with dose. The recombined molecules do not preserve our isotopic HD purity. Some fraction will be ortho- $\mathrm{H}_{2}$ and constitute a growing source of depolarization. In the data so far, we do not see evidence for this mechanism. Within a few hours after the beam ended, the target returned to a frozen spin state with a lifetime too long to determine readily. (see Fig. 5)

\section{Summary}

The target configuration utilized for the 2012 electron beam tests was optimized for initially polarizing the $\mathrm{HD}$ and minimizing the dilution factor, resulting in temperatures around $1 \mathrm{~K}$ with $1 \mathrm{nA}$ of electrons. Changes to optimize instead the cooling under beam should allow B/T to increase by a factor of fifteen. RF shielding is observed and must be taken into account but should not prevent using the HD target with electrons. Consideration of two possible depolarization mechanisms suggests that higher B/T may produce a large improvement in the unacceptably short lifetime seen in the 2012 measurements. In order to test this possibility, we plan to undertake measurements with a new accelerator test facility under development at Jefferson Lab that will provide 5-10 MeV electron beams to our in-beam cryostat.

\section{Acknowledgments}

The authors are grateful to the technical staff of JLab Hall B and to the members of the CLAS collaboration for their aid in switching to and from electron running and providing monitoring of that beam during the test runs. This work has been supported by the United States Department of Energy, Office of Nuclear Physics Division under which Jefferson Science Associates operates Jefferson Laboratory, the US National Science Foundation and the Istituto Nazionale di Fisica Nucleare of Italy. 
Notice: Authored by Jefferson Science Associates, LLC under U.S. DOE Contract No. DEAC05-06OR23177. The U.S. Government retains a non-exclusive, paid-up, irrevocable, worldwide license to publish or reproduce this manuscript for U.S. Government purposes.

\section{References}

[1] www.jlab.org/exp_prog/proposals/06/PR-06-101.pdf.

[2] www.jlab.org/exp_prog/proposals/12/PR12-12-009.pdf \& -010.pdf.

[3] J. A. Forrest and R. L. Brooks, Phys Rev B55 (1997) p906 and references cited therein.

[4] E. Radtke et al., NIM A526 (2004) p168 and references cited therein.

[5] H. Mano and A. Honig, NIM 124 (1975) p1.

[6] A. Honig, Phys Rev Lett 19 (1967) p1009.

[7] X. Wei et al., J. Phys.: Conf. Ser. 400 (2012) p052042.

[8] C. S. Whisnant et al., HD gas purification in proceedings of PSTP 2013, PoS (PSTP 2013) 017.

[9] X. Wei et al., Boosting deuteron polarization in HD targets in proceedings of PSTP 2013, POS (PSTP 2013) 016.

[10] C. D. Bass et al., NIM A737 (2014) p107.

[11] A. L. Woodcraft, Cryogenics 45 (2005) p626.

[12] J. H. Constable and J. R. Gaines, Phys Rev B8 (1973) p3966.

[13] H. E. Rorschach, Jr., Physica 30 (1964) p38.

[14] W. P. Jesse and J. Sadauskis, Phys Rev 97 (1955) p1668.

[15] W. M. Jones and D. F. Dever, J Chem Phys 60 (1974) p2900.

[16] R. W. H. Webeler, J Chem Phys 64 (1976) p2253.

[17] R. W. H. Webeler, private communication in G. Rosen, J Chem Phys 66 (1977) p5423. 